\title{
REVISIONES
}

\section{La investigación sociocrítica en la educación física*}

\author{
Socially critical research in physical education \\ A investigação sociocrítica na Educação Física
}

\author{
José Devís-Devís \\ Universitat de València (España). Telfs.: +34 963983309; 963864351. Correo electrónico: jose.devis@uv.es
}

\section{RESUMEN}

La investigación socialmente crítica en la educación física, aquélla que se realiza con el propósito de contribuir al cambio y la transformación social, se ha visto reconocida durante la última década. Sin embargo, posee una considerable tradición de estudios de este tipo. En este artículo se presentan los antecedentes cercanos y su evolución desde la década de 1980 a la actualidad. Se repasan las principales contribuciones de los inicios y del periodo de internacionalización que vivió este tipo de investigaciones, así como las principales orientaciones y áreas temáticas de interés. También se trazan los principales retos y perspectivas metodológicas y temáticas de futuro. El artículo finaliza señalando la necesidad de este tipo de investigación ante los problemas sociales, económicos y ecológicos de un mundo globalizado e invita a los lectores y las lectoras a participar activamente en el desarrollo de esta tradición investigadora.

Palabras clave: cambio social, educación física, investigación educativa, pedagogía del deporte, teoría crítica, transformación.

\begin{abstract}
Socially critical research in physical education, the one developed in order to contribute to social change and transformation, has been recognized during the last decade. However, it has a considerable tradition of this kind of research. This article provides background and evolution of this type of research from the 1980s to the present. The major contributions of the early period and the internationalization of such inquiries and the main directions and subject areas of interest are presented. The main challenges, as well as the methodological perspectives and issues for the future are also plotted. The paper finishes by noting the need for such research due to the social, economic and ecological aspects of a globalized world, and invites readers to participate actively in the development of this research tradition.
\end{abstract}

Key words: social change, physical education, educational research, sport pedagogy, critical theory, transformation.

\section{RESUMO}

A investição sociocrítica na Educação Física, aquela que se realiza com o propósito de contribuir com as mudanças e transformação sociais, foi reconhecida na última década. No entanto, há considerados estudos anteriores a este período. Apresentam-se os antecedentes mais próximos e sua evolução desde a década de 1980 até a atualidade. É feita um revisão das principais contribuições iniciais e do período de internacionalização de este tipo de investigação, bem como de suas principais orientações e áreas temáticas. Traçam-se também os principais desafios, perspectivas metodológicas e temáticas futuras. Finaliza-se observando a necessidade de tal pesquisa diante dos problemas sociais, econômicos

* $\quad$ Este artículo fue solicitado por la Revista Estudios Pedagógicos en diciembre del 2011 en el contexto del proyecto de Investigación FONDECYT (Fondo de Investigación Científica y Tecnológica) N N $^{\circ} 1110016$, titulado "Educación Física y su función de transformación de las desigualdades sociales: profesorado del área y documentación ministerial”. El artículo fue aceptado en junio de 2012. 
e ecológicos do mundo globalizado e convidando leitores a participar ativamente no desenvolvimento desta tradição investigativa.

Palavras chave: mudança social, Educação Física, pesquisa educativa, pedagogia esportiva, teoria crítica, transformação.

\section{INTRODUCCIÓN}

A lo largo de la historia de la educación podemos encontrar propuestas y prácticas pedagógicas especialmente preocupadas por las desigualdades, la opresión y la justicia social. Se trata de experiencias y proyectos educativos que podríamos denominar como pedagogía crítica. Entre ellos destacan las iniciativas de la primera mitad del siglo XX que comparten la idea de que la acción pedagógica es acción social y, en consecuencia, acción política. Nos referimos, por ejemplo, a la Escuela Moderna de Ferrer y Guardia, la Pedagogía Libertaria de la Escuela de Hamburgo o la Pedagogía del Oprimido de Freire. Sin embargo, en aquella época no podía hablarse todavía de investigación sociocrítica, aunque las experiencias educativas y el pensamiento crítico interactuaron para crear el caldo de cultivo que pergeñara la investigación educativa realizada con el propósito de contribuir al cambio y la transformación social.

Este tipo de investigación emergió durante la década de 1970 como reacción a la ciencia positivista e interpretativa que explicaba y describía cómo era la realidad social, pero que poco o nada hacían por transformarla. Esta ciencia social crítica, como también se le conoce, pretende dar respuesta a los problemas sociales mediante la incorporación de la autorreflexión y la acción de los actores sociales de las comunidades que se estudian y no desde la perspectiva externa y neutral de carácter empírico e interpretativo. Las personas se hacen cada vez más dependientes del conocimiento experto y la ciencia social crítica intenta identificar y desmitificar los patrones de conocimiento y las constricciones sociales que limitan las posibilidades humanas, así como cambiar las condiciones de vida. Para ello, las cuestiones éticas, morales y políticas se interrelacionan con la ciencia y así consiguen orientar a las personas en cuanto a lo que es adecuado y justo en una situación concreta.

En el ámbito concreto de la educación, la investigación sociocrítica pretende ir más allá de la concepción objetivista de la práctica que se apoya en la pesquisa positivista, se interesa por la cantidad de información sobre la práctica educativa y relega al profesorado a meros consumidores de lo que dicta este tipo de investigación. Por esta razón busca formas de conocimiento, más que cantidad de información, que sea profundo y útil. Es decir, conocimiento profundo para comprender las posibilidades/imposibilidades del cambio y facilitar la concientización y también el conocimiento útil para mejorar la práctica educativa.

Ante la tarea de elaborar este artículo y la amplitud que puede adoptar, conviene realizar algunas aclaraciones previas. En primer lugar, no pretendo realizar un trabajo de revisión sistemática de la literatura socialmente crítica en la educación física, aunque inevitablemente recurriré a un volumen importante de publicaciones que considero clave en la evolución de este tipo de investigación. En segundo lugar, el título del artículo parece sugerir que existe un tipo único y determinado de investigación sociocrítica. Sin embargo, se trata de una pluralidad de líneas de investigación que se preocupan por la justicia social, la igualdad y la emancipación de las personas y los grupos humanos. 
Esta diversidad se apoya en perspectivas teóricas diferentes como el marxismo, el neomarxismo y la nueva sociología de la educación, así como en estrategias metodológicas diversas. Incluso el trabajo de los teóricos críticos de la Escuela de Frankfurt, una de las principales aportaciones a la ciencia social crítica, no era un enfoque unificado, a pesar de mantener supuestos compartidos.

Tal vez, la investigación sociocrítica se entienda mejor como una actividad dinámica que se autodefine constantemente por interacción con nuevas aportaciones teóricas, aspecto que a menudo dificulta una clara y aguda diferenciación de la investigación crítica en relación con otras aproximaciones paradigmáticas como la interpretativa, la feminista y la post-estructuralista. Algunas veces resulta difícil catalogar este tipo de investigaciones, por ejemplo, cuando un determinado estudio sigue una metodología etnográfica y utiliza conceptos neo-marxistas para el análisis y la interpretación de los datos o cuando aplica la teoría textual del post-estructuralismo a contextos reales y conflictivos que permiten la acción políticamente transformativa de la investigación crítica. Además, la investigación sociocrítica resulta muy escurridiza, ya que exige tener en cuenta tanto el proceso metodológico como el resultado de la investigación porque el primero debe ser emancipatorio para las personas implicadas y el segundo debe suponer un reto radical en las conclusiones e implicaciones.

\section{LA INVESTIGACIÓN SOCIOCRÍTICA EN LA EDUCACIÓN FÍSICA}

La investigación sociocrítica en la educación física surge durante la década de 1980, muy en conexión con la tradición cualitativa, cuando algunos académicos, la mayoría de ellos de los países de habla inglesa, dirigen la investigación hacia objetivos de igualdad, justicia social y emancipación. Estos autores y autoras se sintieron atraídos por una emergente ciencia social crítica, como le han llamado sus creadores (Fay, 1987; Habermas, 1982), preocupados por lo que era la investigación empírica en un mundo en que la injusticia conforma la vida de las personas y, también, preocupados por cómo producir conocimiento que pudiera ser útil para la práctica emancipatoria. No obstante, las primeras investigaciones fueron precedidas por críticas importantes alrededor de las formas tradicionales de educación física, formación del profesorado e investigación, así como por las correspondientes propuestas alternativas en estos campos.

\subsection{LAS CRÍTICAS A LA ENSEÑANZA Y LA INVESTIGACIÓN EFICAZ}

Las primeras críticas sobre la enseñanza se dirigieron a la planificación técnica del currículum de educación física y la formación efectiva del profesorado. Estas críticas también reclamaban mayor sensibilidad para los problemas sociales y culturales, tales como las desigualdades y la discriminación en la educación física y el deporte. De lo contrario, la profesión de la educación física podría situarse en los límites de la bancarrota moral por desatender, consciente o inconscientemente, dichos problemas (Tinning, 1985). Por otra parte, el profesorado va tomando un papel más activo o, al menos, así se le reconoce desde distintos colectivos, incluido el propiamente docente. El papel reproductor y de comparsa que le asignan los investigadores deja paso a un papel de experto, puesto que se le considera el colectivo que mejor conoce la enseñanza y sus entresijos. Las ideas de 
Stenhouse relativas al profesional 'extensivo' y profesor o profesora como investigadora de sus clases van calando en la educación física (Almond, 1976, 1979, 1985). Es decir, los profesores y profesoras no sólo se dedican a solucionar problemas profesionales sino también a establecer y definir cuáles son los problemas dignos de atención profesional y, por extensión, de investigación (Harris, 1987; Kirk y Smith, 1986; Lawson, 1984). Estas críticas fueron seguidas de propuestas pedagógicas alternativas de distinto tipo y en distintos lugares, pero contenían un sustrato común consistente en convertir a los profesores y profesoras en agentes de cambio social (Hellison, 1978, 1985; Tinning, 1987a).

Las críticas a la investigación positivista dominante enfatizaban las limitaciones de la cuantificación de hechos y comportamientos observables a la hora de comprender y mejorar la educación física y la formación del profesorado (Beamish, 1982; Crum, 1986; Kirk, 1989; Lawson, 1990a). Los objetivos principales de la investigación positivista buscaban comparar y relacionar dichos comportamientos y hechos con otras variables dependientes, generalmente resultados del aprendizaje del alumnado que, en la educación física, consistían inicialmente en el rendimiento conseguido por el alumnado en habilidades o tests físicos (Devís-Devís, 1993, 1996).

Algunos autores iniciaron una discusión abierta con los defensores del paradigma positivista (Schempp, 1987, 1988; Siedentop, 1987) y otros propusieron nuevos paradigmas de investigación para el profesorado y la enseñanza de la educación física (Lawson, 1983a, 1983b, 1985). Fue el reflejo de un debate más amplio en el seno de las ciencias de la educación, conocido como 'la guerra de los paradigmas' (Gage, 1989), sobre la unidad o diversidad epistemológica correspondiente a distintos paradigmas de investigación. Estos paradigmas y nuevos modelos de investigación centrada en el profesorado y el alumnado, algunos de tipo cualitativo, el interés por la socialización profesional y, sobre todo, la búsqueda de conocimiento útil para la práctica de la enseñanza, facilitaron el camino a la investigación sociocrítica.

En la educación física y el deporte, estas discusiones paradigmáticas fueron muy importantes porque abrieron el campo de estudio y contribuyeron a consolidar internacionalmente la investigación pedagógica. Como señalaba Sparkes (1992), esta diversidad debe reconocerse y celebrarse, ya que ofrece diferentes visiones del campo y permite un mayor entendimiento y desarrollo teórico. Según Lawson (1990b: 6), esta situación también ayudó a mejorar la investigación y a preparar su "transición de la adolescencia a la edad adulta". Desde mi punto de vista, el debate paradigmático ha contribuido, al menos, a la comprensión de las preocupaciones y los objetivos de distintas formas de investigación, a aclarar confusiones epistemológicas y a respetar distintos tipos de investigación alrededor de la educación física y la pedagogía del deporte.

\subsection{LOS INICIOS}

Los inicios de la investigación sociocrítica en la educación física, durante la década de 1980, se sitúan en distintos lugares de Europa, América y Oceanía. Las influencias de la Escuela de Frankfurt en Alemania dieron lugar a diversas aportaciones de la pedagogía crítica en la educación física y a unos primeros estudios durante esa década (Landau, 1996). Sin embargo, las investigaciones más influyentes provienen de un grupo de autoras y autores británicos, norteamericanos y australianos, especialmente influidos por la Nueva Sociología de la Educación, la sociología crítica y las teorías neo-marxistas. 
Los textos editados por Evans $(1986,1988)$ reunieron a un número importante de estudios críticos sobre la innovación curricular, la ideología, el sexo y la coeducación. Su principal interés consistía en relacionar lo que pasaba dentro de las clases de la educación física con otros problemas sociales más amplios y profundizar en las relaciones de poder y el control de la asignatura. La micropolítica presente en los procesos de innovación, así como las posibilidades y limitaciones del cambio en las escuelas fueron objeto de atención por parte de Sparkes (1988a, 1988b, 1989).

Los trabajos de Tinning (1987b, 1988) sobre investigación-acción emancipadora representan los primeros intentos de utilizar la investigación con la finalidad de cambiar las prácticas de enseñanza de los estudiantes universitarios de la Universidad de Deakin en Australia, un foco internacional de desarrollo y difusión de este tipo de investigación en la educación y la educación física. Otras contribuciones de Kirk también se ocupan de la formación del profesorado de educación física (Kirk, 1986), las ideologías (George y Kirk, 1988; Kirk y Colquhoun, 1989; Kirk et al. 1986) y el desarrollo pionero de una perspectiva curricular socio-crítica (Kirk, 1988).

Un grupo especial de investigaciones críticas se dirigieron a la educación física de las chicas que mostraban desinterés por esta asignatura. Se trata de los trabajos de la norteamericana Griffin $(1983,1984,1985 a, 1985 b)$ que se centraron en la equidad, el género y la capacidad, así como el poder de los factores contextuales en las clases de educación física (Griffin, 1985c). Los estudios de Scraton $(1986,1987)$ sobre la educación física de las chicas británicas generaron un interés especial por los asuntos de género en Europa, más allá de la teoría de la igualdad de oportunidades, y contribuyeron a comprender la construcción social de la femineidad. También algunos trabajos de Dewar (1987) contribuyeron, desde la sociología crítica y el neo-marxismo, a comprender la construcción social del género en Norteamérica.

Todos los estudios anteriores permitieron que la investigación sociocrítica fuera conocida dentro del campo de la educación física y la pedagogía del deporte. Precisamente, a finales de la década de 1980 aparece la primera revisión de estudios sociocríticos, acompañados de una introducción a las características de este tipo de investigaciones (Bain, 1989). Este tipo de investigación vuelve a ser parte del capítulo que sobre educación física escribió Bain (1990) en un manual internacional sobre la formación del profesorado. Sin embargo, en otras revisiones generales sobre la enseñanza y el currículum de la educación física, este tipo de pesquisa se menciona sólo para indicar que será una perspectiva que requerirá atención en el futuro (Silverman, 1991; Steinhardt, 1992).

\subsection{LA INTERNACIONALIZACIÓN}

La década de 1990 y los primeros años de la década siguiente se caracterizan por la expansión y el desarrollo de las perspectivas socialmente críticas de investigación en la educación física y la formación del profesorado. Un papel destacado tuvieron las revistas internacionales del campo que recogían cada vez más estudios sociocríticos (Quest, Journal of Teaching in Physical Education, European Physical Education Review, Physical Education and Sport Pedagogy y Movimento) y otras nuevas revistas especialmente sensibles a estas cuestiones como la Sport, Education and Society y, posteriormente, Qualitative Research in Sport, Exercise and Health. 
El trabajo iniciado la década anterior se continúa con nuevas aportaciones metodológicas o temáticas. Así, por ejemplo, Sparkes introduce el uso de las historias de vida para dar voz al profesorado y abrir nuevas posibilidades emancipadoras para los profesionales de la educación física, interrelacionando la dimensión personal y profesional de sus vidas (Sparkes, 1994a, 1994b; Sparkes et al. 1990; Sparkes et al., 1993). Por otra parte, Tinning (1992a; Tinning et al., 1996) contribuye, tanto teórica como empíricamente, a la investigación-acción y al desarrollo teórico y la comprensión de los discursos pedagógicos de la formación del profesorado (Tinning, 1991, 1997, 2002). Kirk (1992, 1998a) comienza un periodo en el que se ocupa de la construcción socio-histórica de la educación física escolar y también esboza formas para desarrollar pedagogías críticas informadas por una agenda investigadora que proporcione el conocimiento básico para el cambio emancipatorio en la educación física escolar y la formación inicial del profesorado (Kirk, 2001; Rovegno y Kirk, 1995). A partir de la sociología crítica de la educación y del cuerpo, Evans y colaboradores estudian las relaciones del cuerpo, la salud y la escolarización, resultado de la interacción de diversas teorías sociales, aunque especialmente influido por la nueva sociología crítica europea (Evans et al., 2004).

Los libros colectivos de dimensión internacional incorporan nuevos temas, además de los iniciados con anterioridad, así como puntos de vista diferentes. Entre ellos se encuentran aportaciones influidas por nuevos movimientos culturales y teóricos como un análisis crítico del currículum oculto y la salud o de la pedagogía de la educación física como texto (Kirk y Tinning, 1990). También discusiones y aclaraciones paradigmáticas, estudios sobre el currículum y el género e historias de vida sobre el profesorado y su enseñanza (Sparkes, 1992). La igualdad en la enseñanza y la formación del profesorado, así como las posibilidades/imposibilidades de cambio de las políticas y reformas educativas, fueron temas de atención sociocrítica (Evans, 1993; Penny y Evans, 1999). En ellos ya se observan las relaciones de las teorías críticas con otras aportaciones teóricas y paradigmáticas post-estructuralistas y feministas. Especialmente, se aprecia en nuevos libros colectivos que reflexionan sobre el postmodernismo, sus influencias y retos para la educación física (Fernández-Balboa, 1997a) y en artículos que reflejan todo un programa de investigación post-estructuralista sobre el papel del lenguaje y sus significados en la construcción social del género y las subjetividades en las clases (Wright, 1990, 1995, 1997, 2000, 2004).

El feminismo arraiga entre algunas autoras inicialmente posicionadas en un paradigma crítico que dirigen sus estudios al papel de la educación física en las relaciones de poder y privilegio ligadas al género que existen en las sociedades capitalistas (Dewar, 1990, 1993). Otras se ocupan de estudiar el heterosexismo y la homofobia entre las profesoras lesbianas, así como otros problemas sociales y profesionales ligados a las identidades sexuales no hegemónicas (Clarke, 1996, 1998; Flintoff, 1994; Griffin, 1992a, 1992b, 1998; Squires y Sparkes, 1996; Sykes, 2001). Los conceptos de feminidad y masculinidad hegemónicas se encuentran en la mayoría de trabajos anteriores y son objeto de atención preferente de estudios orientados a las clases de educación física, los recreos y ciertos contenidos como el deporte (Branham, 2003; Chepyator-Thomson y Ennis, 1997; Hickey y Fitzclarence, 1999; Hickey et al., 2000; Light y Kirk, 2000; Renold, 1997).

Como parte del proceso de internacionalización se observa la aparición de estudios de autores de otros países y en otras lenguas, más allá del inglés, debido a la difusión que ejercen las revistas internacionales del campo, la traducción de los libros y documentos 
y al contacto directo con los autores pioneros de la investigación sociocrítica a través de congresos o invitaciones académicas. El análisis de la literatura en otros países y lenguas resulta difícil e inabarcable, pero puedo presentar algunos ejemplos que he tenido ocasión de seguir durante los últimos años. En el caso particular de Brasil, las estancias de algunos profesores en países extranjeros y la creación de cursos de postgrado y doctorado en los que colaboraron académicos del ámbito general de las ciencias sociales y humanas, desde la década de 1980 a la actualidad, han contribuido a la introducción de la pedagogía crítica y la investigación socialmente crítica. Las críticas al deporte y la educación física, así como propuestas pedagógicas críticas orientadas teóricamente por el marxismo del materialismo dialéctico, también sirvieron de punto de arranque a la investigación posterior (Bracht y Caparroz, 2009). No obstante, podemos considerar como primera investigación sociocrítica en la educación física el estudio de casos comparativo entre la asignatura escolar y la cultura física presente en su comunidad extraescolar (Kunz, 1991). Posteriormente, también destacan estudios críticos sobre la cultura profesional y creencias del profesorado de educación física en la ciudad de Porto Alegre (Molina-Neto, 1996; Molina-Neto, 2003) y procesos de investigación-acción para el desarrollo profesional del profesorado de educación física (Bracht et al., 2002; Muñoz, 2004).

En España resultó importante la influencia anglosajona sobre los estudios del currículum de la década de 1980, que afectaron profundamente a la tradición didáctica española del estudio de la educación. Se realizaron muchas traducciones de autores críticos del ámbito de la educación, destacando para la educación física el libro de Kirk de 1988, Physical Education and Curriculum Study: A critical Introduction, el de Scraton de 1992, Shaping up to womanhood. Gender and girls' physical education, y el de Tinning de 1987, Improving Teaching in Physical Education (Kirk, 1990; Scraton, 1995; Tinning, 1992b). Asimismo, fueron muy importantes las relaciones profesionales y las visitas académicas de diversos autores australianos, británicos y norteamericanos mencionados en este capítulo. En este contexto germinaron, unos años más tarde, diversas propuestas, análisis e investigaciones críticas de autores españoles, aunque sin llegar a convertirse en una línea sobresaliente. Así surgieron estudios de investigación-acción realizados con profesorado de enseñanza primaria y secundaria durante largos periodos de tiempo (Fraile y Romo, 1991; López-Pastor et al., 2011) e investigaciones colaborativas que enfatizan el poder del contexto, los facilitadores e inhibidores de la colaboración y la toma de conciencia (concienciación) respecto a problemas y necesidades de cambio en las clases de educación física (Devís-Devís, 1996; Peiró-Velert y Devís-Devís, 1993). También se han realizado estudios sobre las creencias de un grupo de futuros profesores (Martínez, 1994), las ideologías, representaciones sociales y currículum oculto en la educación física (Barbero, 1994, 1996; Devís-Devís et al. 1994; Molina y Beltrán, 2007), la construcción social del género en la asignatura y dificultades de cambio en las relaciones tradicionales de género (González-Ravé et al., 2007; Soler, 2009) y estudios sobre la historia, política y reforma curricular (Barbero, 1993; Devís-Devís, 1997; Hernández, 1996; Molina y Devís-Devís, 2001). Incluso se han publicado artículos que recogen influencias de postulados postmodernos en la investigación sociocrítica, destacando contribuciones sobre las dificultades y la ética en el desarrollo de pedagogías críticas en diferentes aspectos de la enseñanza universitaria (Devís y Sparkes, 1999; Muros y Fernández-Balboa, 2005; SiciliaCamacho y Fernández-Balboa, 2009; Trigueros et al., 2006). Influencias postmodernas también se encuentran en otros libros colectivos con participación de autores españoles 
y extranjeros, orientados a nuevas perspectivas en el estudio y enseñanza de la educación física, el deporte y la salud (Devís-Devís, 2001; Fraile, 2004), a la reflexión crítica del proceso de investigación (Sicilia y Fernández-Balboa, 2004) y a formas alternativas de evaluación en la educación física y la formación del profesorado (López-Pastor, 2006).

En países hispanoamericanos destacan aportaciones relativas a las representaciones sociales e imaginarios de la educación física y su profesorado, especialmente en Colombia (Murcia et al., 2005; Ruiz et al., 2011), pero también en Chile (Almonacid y Matus, s/f) y México (Aguayo, 2009). Algunas investigaciones en este país se han enfocado a proyectos comunitarios de tipo reeducativo e investigación-acción participativa (Aquino et al. 2001; Aquino, 2004) y otros estudios que incluyen las creencias y la violencia verbal y simbólica relacionada con el género en la educación física de primaria (Aquino et al., 2008). El texto colectivo, recientemente publicado en Argentina, también incluye un grupo de contribuciones sociocríticas de autores latinoamericanos y de otros países de habla inglesa sobre la educación física y la formación del profesorado (Martínez y Gómez, 2009).

Con las investigaciones presentadas en este apartado y otras que han podido quedarse en el tintero por limitaciones de espacio, podemos decir que la investigación sociocrítica está reconocida internacionalmente dentro de los estudios dedicados a la educación física, la pedagogía del deporte y la formación del profesorado. La publicación del primer manual internacional de investigación, el Handbook of Physical Education en el que se incluye un capítulo marco sobre las perspectivas sociocríticas de investigación (Devís-Devís, 2006), es el mejor ejemplo de su reconocimiento como una de las grandes tradiciones investigadoras en este campo de estudio.

\subsection{LAS PRINCIPALES ORIENTACIONES Y TEMÁTICAS DE INVESTIGACIÓN}

El apartado anterior aporta una introducción a la investigación sociocrítica con enfoque histórico, pero resulta necesario complementarlo con las principales orientaciones de investigación, así como con un mapa temático general de los estudios realizados internacionalmente.

Las principales orientaciones de pesquisa sociocrítica en la educación física, de acuerdo con su principal objetivo, corren paralelos al conjunto de la investigación educativa y pueden concretarse en dos: a) análisis y crítica sociocultural; e b) investigación participativa o dirigida al empoderamiento.

Para algunos autores, el objetivo principal de este tipo de investigación es la crítica social y cultural de las ideologías y las relaciones de poder. Pero este propósito no sólo consiste en identificar y exponer las ideologías y las relaciones de poder, sino que su conocimiento empape la conciencia de los oprimidos para que provoque la acción social y les ayude en su auto-emancipación (Carr y Kemmis, 1988). Por lo tanto, la crítica no sólo permite comprender lo encubierto, lo evidente o dado por supuesto alrededor de la educación física y el deporte, sino a actuar positivamente para el mejor futuro posible.

En cambio, otros autores están más preocupados por el potencial de empoderamiento (o capacitación) de la investigación crítica con el fin de aumentar la capacidad de los participantes, individuos o grupos, para tener un mayor control de sus decisiones y poner en entredicho las relaciones y estructuras de poder. Se trata de borrar la separación entre la investigación y la acción mediante la combinación de la educación, la acción 
socio-política, y la investigación (Fals-Borda, 1992). Debido a un uso amplio del término empoderamiento, cabe destacar que puede tomar muchas formas y no sólo se producen a nivel de la conciencia individual, sino también a los niveles sociales y estructurales.

Recientemente, en el campo de la educación física, Kirk (2001) ha afirmado que la auto-conciencia es un aspecto necesario del empoderamiento aunque no suficiente en sí mismo, ya que requiere un cambio social. Por otra parte, el empoderamiento no es algo que se transmite de una persona a otra, sino una experiencia compartida donde el poder aumenta y se redistribuye entre los participantes para tener un mayor control de sus vidas. Como proceso, el empoderamiento implica escuchar y comprender las condiciones sociales, dialogar para identificar los problemas de investigación y acabar con la acción social para cambiar esos problemas (Freire, 1973, 1987).

Estos dos grandes tipos de investigación atraviesan distintas temáticas que preocupan a los investigadores e investigadoras sociocríticos y convendría conocer el mapa temático para comprender la amplitud de problemas que han tratado a lo largo del tiempo. La Tabla 1 muestra los principales temas y subtemas, así como los intereses y contenidos vinculados a dichos subtemas. Por esta razón, me ocuparé de las características y grandes temas sociocríticos, explicando por qué son preocupaciones de los investigadores y evitando ser repetitivo con la información contenida en la tabla. Estos grandes temas son, según Devís-Devís (2006) y otras aportaciones de este trabajo: 1) la construcción social de la educación física y la formación del profesorado; 2) la socialización profesional; y 3) la política y la práctica en la profesión.

El primer gran tema de interés investigador recoge estudios diversos derivados de considerar la educación física y la formación del profesorado como productos históricos que están conformados por las cambiantes fuerzas políticas, sociales, económicas y culturales, así como educativas. Para los investigadores sociocríticos, estos dos contextos de actividad profesional son el legado de los enfrentamientos y luchas entre grupos rivales. Es decir, la educación física no se ha construido por la acumulación de aportaciones continuas heredadas a lo largo del tiempo, sino que se ha ido conformando como resultado de las pugnas entre colectivos diferentes de personas que entienden de manera distinta la asignatura, sus prácticas y funciones. Por ello, los investigadores se han ocupado de los significados, valores, creencias, interacciones y prácticas presentes en dichos contextos, ya que en estos temas descansa el potencial para reproducir o cuestionar las relaciones de poder en la profesión. Por lo tanto, los estudios socio-históricos y los análisis de las ideologías y relaciones sociales emergen como importantes áreas de pesquisa. 


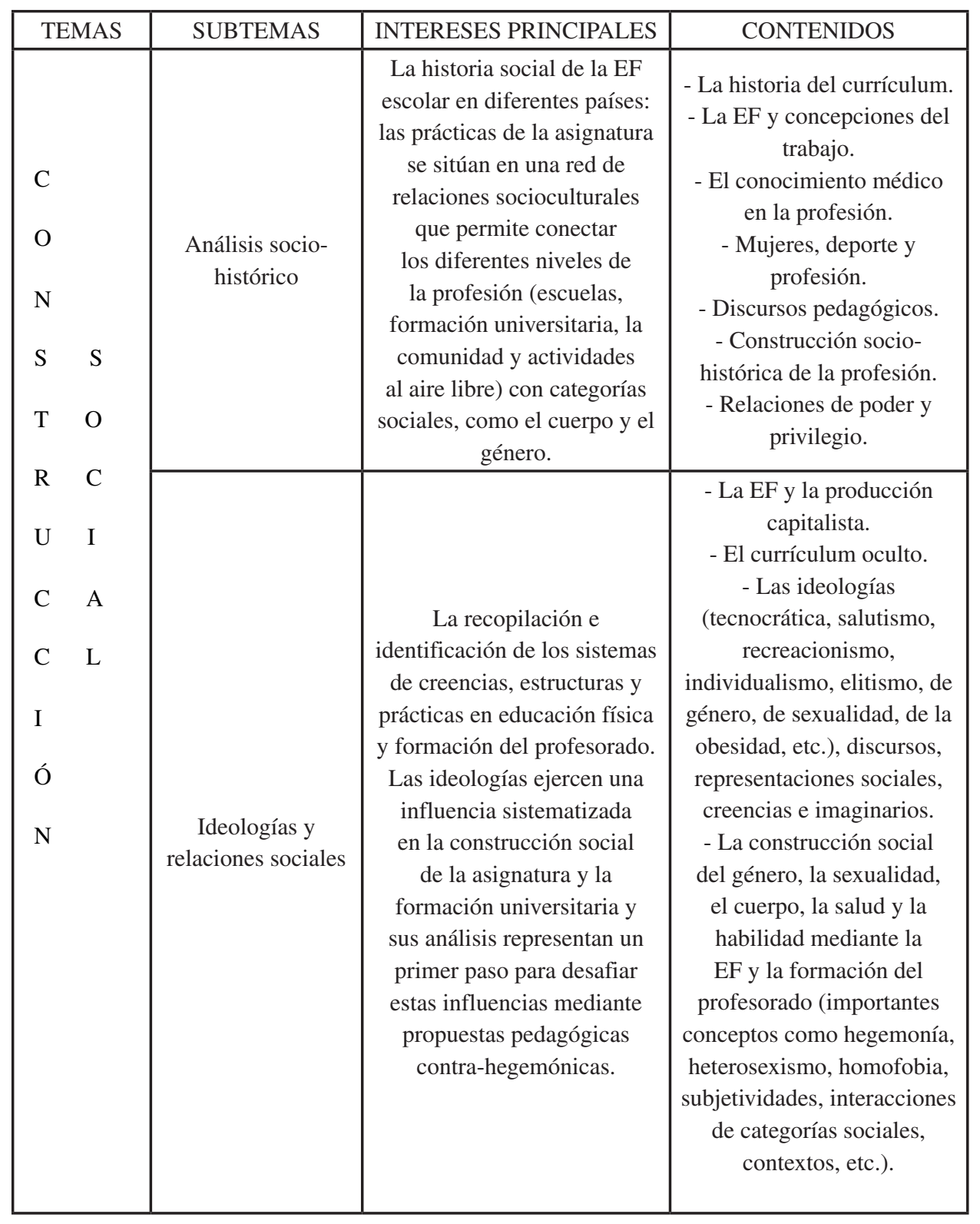




\begin{tabular}{|c|c|c|c|c|}
\hline \multicolumn{2}{|l|}{$\mathrm{S}$} & $\begin{array}{l}\text { Reclutamiento } \\
\text { previo }\end{array}$ & $\begin{array}{l}\text { Importancia significativa en } \\
\text { la formación temprana de las } \\
\text { creencias y valores relativos } \\
\text { al trabajo del profesorado. }\end{array}$ & $\begin{array}{l}\text { - Escasa atención de la } \\
\text { investigación sociocrítica. }\end{array}$ \\
\hline $\mathrm{O}$ & $\mathrm{P}$ & \multirow{5}{*}{ Formación inicial } & \multirow{5}{*}{$\begin{array}{l}\text { Las creencias y los valores } \\
\text { iniciales de los estudiantes } \\
\text { permanecen, en gran parte, } \\
\text { sin cambios durante toda } \\
\text { la formación inicial del } \\
\text { profesorado. }\end{array}$} & \multirow{5}{*}{$\begin{array}{c}\text { - Análisis críticos } \\
\text { de programas } \\
\text { universitarios (utilitario, } \\
\text { tecnocrático, sexista, } \\
\text { desprofesionalización, } \\
\text { aislamiento profesional, } \\
\text { etc.) } \\
\text { - Dificultades, emociones } \\
\text { y tensiones en enseñanza } \\
\text { sociocrítica. }\end{array}$} \\
\hline C & $\mathrm{R}$ & & & \\
\hline A & $\mathrm{F}$ & & & \\
\hline $\mathrm{L}$ & $E$ & & & \\
\hline I & $\mathrm{S}$ & & & \\
\hline Z & $\mathrm{O}$ & \multirow{7}{*}{$\begin{array}{l}\text { Trabajo docente } \\
\text { y carreras } \\
\text { profesionales }\end{array}$} & \multirow{7}{*}{$\begin{array}{l}\text { Relacionar las identidades } \\
\text { del profesorado con los } \\
\text { contextos en que se localizan } \\
\text { y construyen. De este modo, } \\
\text { existen más posibilidades } \\
\text { para contribuir a que el } \\
\text { profesorado se convierta en } \\
\text { agente de cambio social. }\end{array}$} & \multirow{7}{*}{$\begin{array}{c}\text { - Principiantes y } \\
\text { experimentados. } \\
\text { - Condiciones de trabajo } \\
\text { docente. } \\
\text { - Identidades profesionales } \\
\text { y personales y sus } \\
\text { interacciones. } \\
\text { - Cambios corporales y } \\
\text { pedagogía. } \\
\text { - Micropolítica del trabajo } \\
\text { docente. } \\
\text { - Proletarización del } \\
\text { profesorado. } \\
\text { - Marginalización } \\
\text { profesional. }\end{array}$} \\
\hline A & $\mathrm{R}$ & & & \\
\hline C & $A$ & & & \\
\hline I & $\mathrm{D}$ & & & \\
\hline & $\mathrm{O}$ & & & \\
\hline $\mathrm{N}$ & & & & \\
\hline & & & & \\
\hline
\end{tabular}




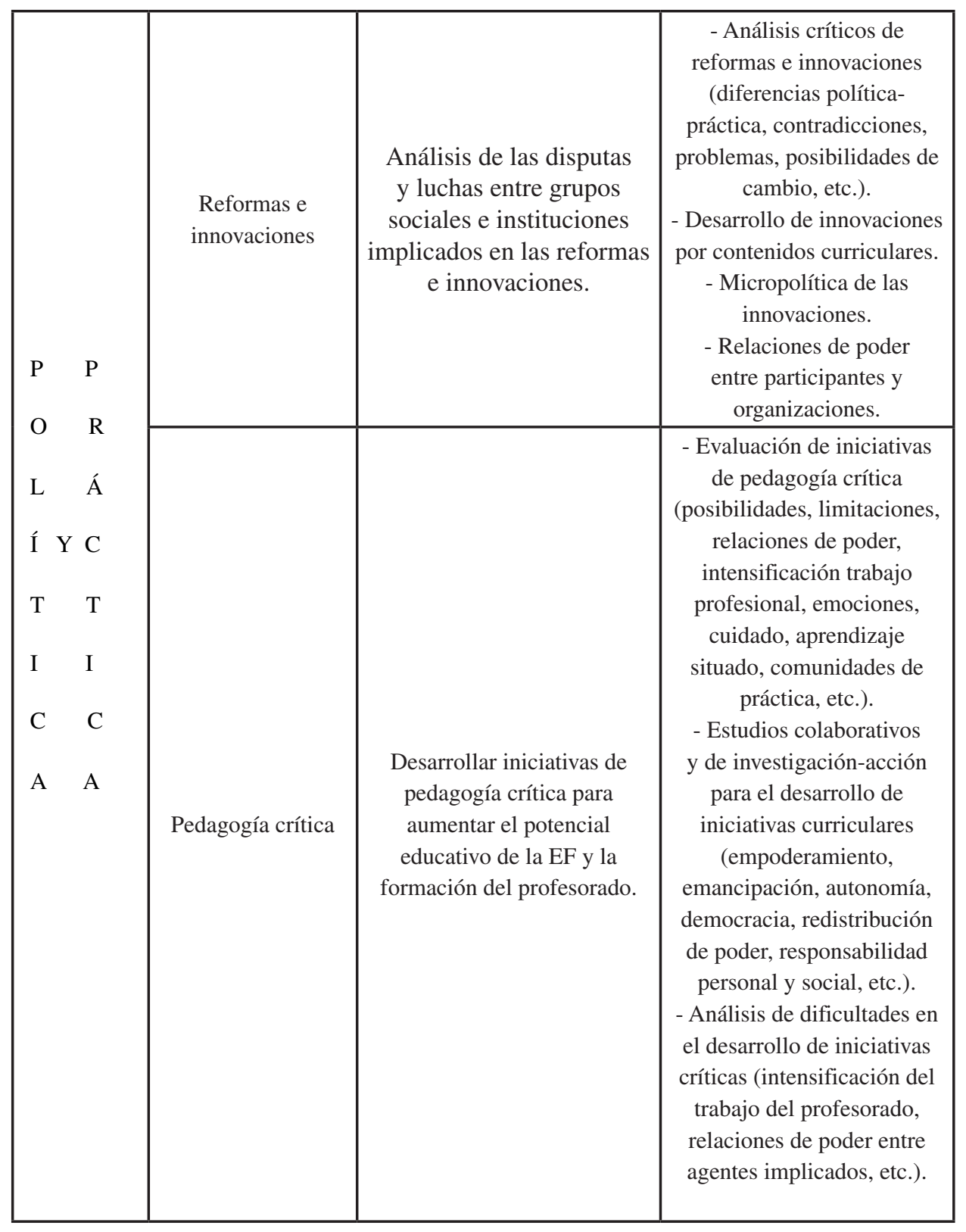

Tabla 1. Aproximación temática a la investigación sociocrítica 
El segundo gran tema se refiere al proceso subjetivo, dialéctico y prolongado de la socialización profesional que, desde una perspectiva sociocrítica, también está histórica y culturalmente construido. Es decir, los significados hegemónicos y los valores de la profesión de la educación física son el resultado de la negociación constante de los profesionales con los condicionantes socioculturales con los que conviven a lo largo de su vida. Por lo tanto, entre los temas importantes para los investigadores sociocríticos se encuentran los significados, valores, relaciones y situaciones presentes en su proceso de socialización, desde el tiempo en que asistieron a las clases de educación física en las escuelas hasta que vuelven a ellas como profesores y profesoras. Eso incluye, también, el tipo de acceso a la formación inicial, la manera y situación en que se forman, la características del acceso a la profesión, las circunstancias de su trabajo, las relaciones con otros profesionales, agentes e instituciones, las relaciones entre lo personal y lo profesional, así como el desarrollo y evolución de sus carreras profesionales. Y todo ello, obviamente, sin perder de vista los principales objetivos de este tipo de investigación que he señalado al comienzo de este apartado.

El tercer gran tema gira alrededor de las políticas y las prácticas educativas. Las políticas son guías generales o propuestas para ordenar los asuntos educativos, mientras que las prácticas son acontecimientos reales que dependen de la articulación compleja e incontrolable de las relaciones que se producen dentro y fuera de un sistema educativo. Entre la política y la práctica surgen conflictos y contradicciones que indican la existencia de relaciones de poder, control e ideología, naturalmente en el marco de contextos socio-históricos determinados. Dos grandes grupos de estudios surgen dentro de esta gran temática; una dirigida a las reformas e innovaciones y otra, específicamente, a las iniciativas de pedagogía crítica. En la primera predomina la crítica sociocultural porque las reformas y las innovaciones son espacios estratégicos que están abiertos a los intereses y las disputas de diversos grupos e instituciones sociales. Son procesos sociales de cambio de carácter complejo, incontrolable y, en gran medida, desconocido que evolucionan en conexión con las instituciones sociales y los modos en que una sociedad se organiza y controla. En la segunda predomina la investigación participativa para el desarrollo de iniciativas de pedagogía crítica o identificar problemas para su desarrollo, tanto en la educación física escolar como en la formación del profesorado.

\subsection{LOS RETOS Y LAS PERSPECTIVAS DE FUTURO}

El carácter dinámico de la investigación sociocrítica, mencionado en la introducción de este artículo, es la respuesta a las necesidades marcadas por los cambios sociales y a las críticas, internas y externas, realizadas a ciertos postulados teóricos o actitudes de investigación. Esta respuesta se ha concretado en propuestas de revisión y ampliación de las preocupaciones sociocríticas que se han dirigido alrededor de la ética del cuidado, el aprendizaje del estudiante, la cultura física, la responsabilidad personal y social y los asuntos ecológicos en su relación con la educación física y el deporte (Fernández-Balboa, 1993; Kirk, 2001; Rovegno y Kirk, 1995).

Esta ampliación de la agenda sociocrítica no ha estado exenta de discusión y autocrítica. Algunos autores, que realizan su actividad investigadora dentro de esta tradición, manifiestan que dicha ampliación puede confundir el foco de atención singular de este tipo de investigación, es decir, su carácter político y emancipador (Tinning, 2002). Sin 
embargo, las mayores críticas provienen de posiciones externas procedentes del empirismo, el feminismo y post-estructuralismo. Así, por ejemplo, las empiristas han criticado el carácter escurridizo, provocativo, poco fundamentado e, incluso, ofensivo que adopta, a menudo, la investigación sociocrítica (O'Sullivan et al., 1992). Las feministas y postestructuralistas critican la abstracción teórica, la falta de conexión con las prácticas y el olvido de la multiplicidad, diferencia y particularidad en sus estudios de carácter global (Ellsworth, 1989). También han criticado el enfoque dualista de las relaciones de poder en lugar de considerarlo como un fluido, al modo foucaultiano, el peligro de caer en la dominación cuando se pretende el empoderamiento, debido a que se parte de una posición de verdad o la ausencia de un análisis del proceso de estudio en el que se encuentre el propio investigador (Gore, 1992, 1996).

Estas críticas han informado y provocado nuevos retos para el presente y el futuro desarrollo de la investigación sociocrítica, aunque no sean compartidas absolutamente. Los teóricos e investigadores han tomado de estas críticas aquello que puede sumar y estar al servicio de la transformación social y la acción política (Kincheloe y McLaren, 2000). Así, por ejemplo, se introducen nuevos conceptos como la diferencia, la particularidad, el lenguaje y la subjetividad, junto con las categorías sociales de clase, género, raza, edad o lugar. Otros más tradicionales como, por ejemplo, el concepto de ideología se ve afectado de tal manera que no sólo se refiere al sistema de creencias y prácticas sociales, sino también al lenguaje y discursos que contribuyen a la construcción de simbolismos colectivos.

\subsubsection{Las nuevas preocupaciones teórico-metodológicas}

Algunas de las críticas anteriores, como la posición de verdad (régimen de verdad) que se adoptan en muchas investigaciones, han provocado propuestas de superación con una buena dosis de 'humildad investigadora' (Kincheloe y McLaren, 2000). Esto significa aceptar que la emancipación es un proceso que nunca termina, que la investigación social crítica puede resultar falible y que cualquier intento de emancipación no garantiza un resultado equivalente. Además, desde que la investigación está histórica y geográficamente situada, puede que lo que es emancipador en una situación no lo sea tanto en otra. Esta humildad también se refiere a la manera en que investigadores críticos analizan e interpretan los datos empíricos, ya que deben reconocer su dependencia de los marcos teóricos elegidos y sus supuestos ideológicos individuales y sus biografías. Finalmente, esto también significa asumir que no existen sistemas de explicación dominante sino todo un conjunto de perspectivas e interpretaciones socioculturales de los fenómenos sociales. En la educación física, el deporte y la formación del profesorado destacan las reflexiones de Tinning (2002) sobre la 'modestia' de la pedagogía crítica ante los problemas y dificultades de su desarrollo.

La preocupación existente en las últimas décadas, dentro de las ciencias sociales, por las formas de representación y las narrativas también se ha observado entre los investigadores sociocríticos de la educación física y el deporte. Es una contribución postmoderna que busca nuevas formas narrativas, más allá de relatos realistas y neutrales, para evitar que los autores parezcan ausentes de las pesquisas y textos elaborados por ellos y ellas, asî como de los informes globales con el común denominador de los participantes y la voz omnipresente del investigador o investigadora. Se trata de enriquecer las formas realistas 
con otras que incluyen más información de cómo se ha realizado el trabajo de campo, el papel del investigador en el proceso de investigación, distintas voces participantes y formas más abiertas de narración. Además, intentan acercarse al lector y lectora mediante un lenguaje más ordinario y entendible, con el uso de diferentes recursos literarios como la poética, la metáfora, el humor y la ironía. Para los investigadores sociocríticos resulta vital que el texto del relato con el que representan sus estudios capte la atención de los lectores y lectoras porque en ello descansa la capacidad de alterar sus conciencias y el potencial transformador (Foley, 1992). Las contribuciones más relevantes, tanto teórica como empíricamente, proceden de Sparkes y colaboradores que se han ocupado de las narrativas, algunas de ellas dirigidas a propósitos socialmente críticos (Sparkes 1997, 2002a, 2002b, 2009, Sparkes et al. 2003,; Sparkes y Smith, 2008). En el contexto español también se han realizado aportaciones teóricas (Pérez et al. 2011a, 2011b; Sparkes y Devís-Devís, 2007) y empíricas sobre nuevas formas narrativas de tipo biográfico y autobiográfico en la formación del profesorado (Devís-Devís y Sparkes, 1999, 2004; Sicilia, 2011).

Las cuestiones de validez y reflexividad, a menudo conectadas con los retos de la representación, son vitales para el futuro de la investigación sociocrítica. Además de las formas de validez que se utilizan en el paradigma interpretativo, la investigación emancipatoria u orientada a la práctica busca formas particulares de validez más acorde con los propósitos de este tipo de pesquisa. Lather (1991) propuso la denominada 'validez catalítica' que se refiere al grado en que el proceso de investigación orienta y facilita a los participantes la alteración de su conciencia para conocer la realidad y transformarla mejor.

Este tipo de validez es similar al criterio de 'eficacia', desarrollado por autores de la investigación-acción participativa, utilizado en el cambio de las prácticas sociales (McTaggart, 1997; Zamosc, 1992). De acuerdo con los dos propósitos de la investigación sociocrítica, este criterio de eficacia debe utilizarse, tanto a nivel de las representaciones ideológicas como al nivel político (Zamosc, 1992). En el primer nivel la eficacia se produce cuando los actores perciben y comprenden los factores que operan en su propia realidad, de tal manera que pueden emerger nuevas actitudes y valores. En el segundo nivel, la eficacia se espera que produzca cambios en la situación real de los actores para llegar a sus metas colectivas. El problema estriba, señala Zamosc, en saber hasta qué punto los cambios ideológicos y políticos se deben a la investigación o a otros factores que influyen en ella. Como no es posible el control técnico en este tipo de investigación naturalista, la validez depende, en última instancia, de la confianza en la integridad de los investigadores, su autocrítica y reflexividad. Este último concepto consiste en el proceso de reflexión crítica dirigida, tanto al conocimiento producido por la propia investigación como a la manera con que se genera dicho conocimiento. Smyth y Shacklock (1998) lo ven como una revelación de las creencias del investigador, sus valores e intereses, y una estrategia de cuestionamiento de sí mismo. También lo entienden como un proceso deliberado en busca de las tensiones y contradicciones que aparecen en la práctica de la investigación, así como a ser conscientes de la propia complicidad de los investigadores en lo que critican.

Recientemente, Dadds (2008) menciona la 'validez empática' con la que se refiere a la influencia que la investigación sociocrítica, en concreto la investigación-acción, puede tener en las personas implicadas (interna) y en audiencias externas a las participantes en el propio proceso de pesquisa (externa). Se apoya en el potencial que posee este tipo de 
investigación, ya sea en el proceso como en sus resultados, para la transformación de las disposiciones emocionales entre las personas, de tal manera que se crean sentimientos positivos entre ellos. Se trata de conseguir una mayor empatía o capacidad para sintonizar emocionalmente con los demás. Según Goleman (1999), esta capacidad de las personas se asienta sobre la conciencia emocional de uno mismo, puesto que cuanto más abierta se encuentre una persona a sus emociones más destreza adquirirá en la comprensión de las emociones de los demás. Y la clave para acceder a las emociones ajenas parece situarse en la habilidad para captar los mensajes no verbales. En cualquier caso, como señala Dadds (2008), la empatía permite conectar el conocimiento de los participantes y de otros más allá de la propia investigación y aprender de ella. Por otra parte, dadas las relaciones de la empatía con el altruismo y los juicios morales (tienen que ver con víctimas potenciales), este tipo de validez vuelve a descansar en el compromiso moral de los investigadores y participantes en la investigación sociocrítica, así como la reflexividad y autocrítica señalada más arriba.

Por último, quisiera apuntar un elemento fundamental de la investigación sociocrítica si pretende alcanzar los objetivos por los que adquiere su sentido y legitimidad (la crítica sociocultural y la transformación social). Me refiero a la continuidad de las pesquisas y procesos colaborativos y autocríticos en que se sustenta este tipo de investigación. Al igual que ocurre con los procesos de transformación social en contextos deprimidos y vulnerables, la investigación sociocrítica no puede limitarse a aportaciones puntuales, desconectadas de redes o programas de investigación. Sus objetivos no se verán colmados si no existe continuidad, si los procesos individuales no dan paso a procesos de investigación colectiva donde resulta fundamental la colaboración a lo largo del tiempo. En este sentido, es importante no quedarse, como señala Lawson (1991), en investigaciones colaborativas individualistas. Es decir, en iniciativas puntuales, sin gran continuidad, con pocos miembros en el grupo de colaboración, sin apoyo institucional ni sistema de recompensas que lo facilite, sin compartir los resultados y las experiencias con otros colectivos o audiencias y pudiendo acabar únicamente con la lectura de una tesis doctoral. Por eso, autores destacados del ámbito de la educación han evolucionado de un énfasis metodológico a otro más político ligado a la construcción de comunidades críticas de investigadores e investigadoras y/o profesorado comprometidos con la mejora de sus prácticas a través de la investigación acción emancipadora (Kemmis, 1992).

\subsubsection{Los nuevos temas y áreas de interés}

Los cambios sociales, las críticas y los avances sociocríticos han procurado nuevos temas y áreas de interés para la investigación que se caracterizan por mayor complejidad y profundidad en ciertos aspectos que han pasado desapercibidos a los investigadores e investigadoras. Entre ellos podemos distinguir:

- Las voces de los estudiantes. Aunque un grupo inicial de estudios recogieron la voz de los estudiantes desde la perspectiva liberal del paradigma mediacional del pensamiento de los estudiantes, han ido apareciendo diversas contribuciones desde un punto de vista socialmente crítico. Una de las primeras contribuciones se ocupó del papel de los estudiantes en la construcción de un proyecto innovador en la educación física (Brooker y Macdonald, 1999). Especialmente interesante resulta el estudio realizado con estudiantes con severas dificultades de aprendizaje dentro de un programa de nueve sesiones en el 
que sus opiniones y experiencias contribuyeron a desarrollar el currículum (Fitzgerald et al. 2003). Las experiencias negativas de los estudiantes han sido exploradas a través de sus voces con el apoyo de conceptos como la cultura de la performatividad y la violencia simbólica para comprender cómo en un contexto de promoción de la actividad física se acaba favoreciendo la inactividad (Beltrán-Carrillo et al. 2012). Aun así, son necesarios más estudios para conocer cómo los estudiantes contribuyen en la construcción social de la educación física y formación del profesorado y el papel que juegan en la reproducción y transformación de formas de pedagogía crítica. Precisamente el texto colectivo de O'Sullivan y MacPhail (2010) trata de ocupar este vacío de las voces y las experiencias de los estudiantes en los contextos de la educación física y el deporte.

-Los aprendizajes de los estudiantes. Esta área de investigación ha despertado un creciente interés en los últimos años y se basa en la idea de que la capacidad de pensar y actuar críticamente requiere el desarrollo de una teoría aplicable tanto al aprendizaje del alumnado en la formación del profesorado como de la escuela (Kirk, 2001). La literatura sugiere que el aprendizaje situado puede proporcionar avances teóricos para la investigación empírica de tipo sociocrítico y promover pedagogías que facilitan el pensamiento creativo (Kirk y Macdonald, 1998; Rovegno y Kirk, 1995). De estas posiciones teóricas, el aprendizaje se considera multidimensional, y los estudiantes se consideran agentes activos de su aprendizaje. Estudios recientes están utilizando estos métodos en la educación física escolar, más específicamente en el contexto de la enseñanza para la comprensión en los juegos deportivos y unidades deportivas de educación (Kirk y MacPhail, 2002; MacPhail et al. 2008).

-Las interacciones entre categorías sociales. La mayor parte de la literatura que trata de la construcción social de la educación física se centra en una categoría mediacional de análisis, como la clase social, el género o la raza. En consecuencia, existe la necesidad de reconocer e investigar la complejidad social asociada a las interacciones entre raza, cultura, clase social, ubicación, género, edad, religión, capacidad y sexualidad que crean las subjetividades del alumnado y el profesorado (Flintoff et al. 2008). De la misma manera, existe la necesidad de indagar en la opresión y desigualdades que se ocultan entre las interacciones de estas categorías. Este es el caso del estudio que se ocupa de los vínculos intergeneracionales con la reproducción de la masculinidad y la feminidad (Brown y Evans, 2004) o el impacto que la ubicación, el género y la cultura juegan en el acceso y el compromiso de los estudiantes a las oportunidades de práctica física (Wright et al. 2003). Algunos trabajos se han realizado a partir de la combinación de género y raza para conocer las experiencias en la educación física y la interacción religión-educación de las chicas musulmanas que viven en países occidentales (Benn et al. 2011; Dagkas y Benn, 2006).

-La construcción social de los cuerpos y capacidades/discapacidades. Como una parte del ser humano, el cuerpo está en todas partes, en medio de cualquier interacción social e identidad. Probablemente no hay mejor lugar para ilustrar el 'yo' que en los cambios corporales. Sin embargo, hasta hace poco, los investigadores sociocríticos no han prestado atención al papel que el deporte escolar y la educación física juegan en el proceso de construcción social del cuerpo, las identidades corporales y sus relaciones con la salud. El libro editado por Evans, Davies y Wright (2004) y el número monográfico de la revista Sport, Education and Society, coordinado por Evans y Davies (2011), son ejemplos del estudio de la construcción social del cuerpo y la corporalidad, realizados 
desde diferentes perspectivas teóricas como la neo-marxista, la Nueva Sociología de la Educación, la feminista y la postestructuralista. Por lo que se refiere a la capacidad/ discapacidad, los estudios de construcción social comienzan a recoger evidencia de la misma y se espera que aumente en el futuro (Fitzgerald, 2005; Hay y Macdonald, 2011)

-Interacciones entre contextos sociales. El conocimiento en el campo de la educación física y el deporte se construye en muchos sitios diferentes, como escuelas, parques, universidades, medios de comunicación, y así sucesivamente, y los estudios de crítica social se encuentran principalmente en uno u otro de estos lugares. Por lo tanto, para entender cómo los discursos de educación física y el deporte se construyen, transmiten y transforman dentro de los entornos complejos y contextos antes mencionados, las indagaciones deben dirigirse a las interacciones entre dichos contextos. De especial interés son las relaciones dialécticas y las influencias mutuas entre las universidades y la educación física, las profesiones de ejercicio y el deporte para atender las necesidades de la comunidad, especialmente de las personas desatendidas por estas profesiones que tradicionalmente se han centrado en las personas con talento, capaces, habilidosos y en forma (Lawson, 1999). Un ejemplo reciente de esta nueva tendencia explora las interacciones escuela-universidad usando, de manera complementaria, los marcos teóricos de la socialización ocupacional, enculturación en las comunidades de práctica y la teoría de Bernstein sobre el discurso pedagógico (Macdonald et al. 1999). La teoría del dispositivo pedagógico de Bernstein (1998) es especialmente relevante aquí porque permite relacionar distintos contextos o campos como los de producción (p. ej. universidades) y reproducción (p. ej. escuelas) o los de recontextualización oficial (p. ej. administración educativa) y pedagógico (p. ej. profesorado). Así se ha evidenciado, por ejemplo en el estudio sobre la selección de los materiales curriculares en la educación física española (Devís et al. 2011).

- La cultura popular y la educación física. Otro grupo de investigaciones de reciente interés se ocupa de las relaciones entre la educación física escolar y la cultura popular porque los materiales culturales que dan sentido a la educación física (deporte, ejercicio, juego) se encuentran fuera de la escuela. Para evitar el aislamiento del programa escolar de la sociedad en la que están inmersos es necesario comprender los efectos e influencias de la cultura popular en los estudiantes y sus visiones de la educación física (Fernández-Balboa, 1997b). Por lo tanto, es necesaria la comunicación dialéctica entre la educación física escolar y la cultura física para fortalecer formas de transformación social que vayan más allá de las cuatro paredes de las escuelas (Kirk, 1998b; Macdonald, 2003). Por ejemplo, el estudio comparativo de casos de Kunz (1991) analizó las tensiones, complementariedades y funciones entre los estudiantes de educación física en prácticas y su cultura física en dos escuelas brasileñas, y propuso un plan de estudios a partir de estos datos empíricos. Otro proyecto de investigación, desarrollado por Tinning y Fitzclarence (1992) en Australia, estudió las relaciones entre la educación física, los medios de comunicación y las vidas de los estudiantes para implicarse como consumidores críticos de actividad física fuera del sistema escolar. La investigación reciente se apoya en el concepto de 'comunidades de práctica' para comprender y desarrollar estrategias y programas sociales que fomenten conexiones entre la educación física y la cultura popular (Kirk y Macdonald, 1998). Este concepto es tan flexible que permite abordar estudios no sólo para conectar contextos sino también identidades y culturas como ha realizado Paechter (2003) al considerar las masculinidades y feminidades como comunidades de práctica. También se ha utilizado para comprender cómo funcionan los departamentos de 
educación física en las escuelas cuando reciben estudiantes universitarios de educación física (Sirna et al. 2008).

- Ética del cuidado y la responsabilidad. Han aparecido nuevas áreas de investigación alrededor de la emoción y el cuidado de los seres humanos y el medio ambiente, debido a que la ética del cuidado y la responsabilidad se ha propuesto como la base moral para el trabajo sociocrítico, además de la ética más tradicional de la justicia y la emancipación. Un proyecto reciente de investigación cualitativa ha estudiado el rol que la comprensión emocional juega en la enseñanza de la educación física y su influencia en el contenido, la pedagogía y el currículo (McCaughtry, 2004). La dimensión emocional también emerge en un estudio biográfico centrado en la crisis de identidad de un estudiante universitario que se produjo en el contexto del desarrollo de una pedagogía crítica en formación del profesorado (Devís-Devís y Sparkes, 1999). También se ha identificado los problemas emocionales implicados en un proyecto colaborativo de innovación (Devís-Devís y Antolín, 2001), el papel de las emociones en las relaciones de género en la formación del profesorado (Dowling, 2008) y se ha favorecido la comprensión emocional en las prácticas de estudiantes universitarios de educación física (Klemola et al. in press). El cuidado también ha sido un concepto central de algunas profesoras e investigadoras en su trabajo con las adolescentes desinteresadas en la educación física y que pretenden cambiar esa situación transformándola (Ennis, 1999; Ennis et al. 1997). Este trabajo se relaciona con el modelo de responsabilidad social de Hellison, desarrollado para trabajar con jóvenes en situación de riesgo, cuya efectividad ha sido empíricamente apoyado en los EE.UU. y en otros lugares para promover los valores personales y sociales (Debusk y Hellison, 1989; Escartí et al. 2005; Escartí et al. 2010; Hellison y Walsh, 2002; Wright et al. 2004). Las pedagogías de la responsabilidad están comenzando a estudiarse para explorar el impacto humano (es decir, deporte, ejercicio, actividades recreativas) sobre el medio ambiente (Lake et al. 2001). Debemos tener en cuenta que la ética siempre se había dirigido a los asuntos humanos, pero debido al poder de actuación de los seres humanos sobre el planeta y la creciente vulnerabilidad de la naturaleza, la ética del cuidado y la responsabilidad se ha extendido a estos asuntos. La educación física y el deporte implican actuaciones sobre la naturaleza que han pasado desapercibidas y la extensión de la ética del cuidado y la responsabilidad las sitúa en un primer plano.

\section{COMENTARIOS FINALES}

En este artículo he presentado la investigación socialmente crítica, realizada en el ámbito de la educación física y la formación del profesorado, desde sus inicios en la década de 1980, con el propósito de presentar la evolución de la misma, los temas, los autores y las autoras, así como los retos y las perspectivas de futuro a nivel metodológico y temático. Estos retos y perspectivas marcan, a mi entender, los caminos por los que transitará la nueva investigación con la que contribuir a una sociedad más justa, democrática, equitativa, segura y responsable.

Como he señalado en otra ocasión, con los problemas sociales, económicos y ecológicos de un mundo globalizado, la investigación sociocrítica y su acción política no sólo son intelectualmente emocionantes y desafiantes, sino también necesarias. Por último, 
con el artículo pretendo estimular a los lectores y las lectoras a participar activamente en el desarrollo de esta perspectiva o tradición investigadora.

\section{REFERENCIAS BIBLIOGRÁFICAS}

Aguayo, B. (2009). Medios de comunicación y corporeidad: representaciones sociales de los educadores físicos. Comunicación presentada en el X Congreso Nacional de Investigación Educativa. Veracruz, Veracruz.

Almonacid, A. y Matus, E. (sin fecha). La clase de educación física, una indagación desde el imaginario de los estudiantes de pedagogía en educación física de la Universidad Autónoma de Chile- sede Talca. Investigación para el grado de Magister. Universidad Autónoma de Chile.

Almond, L. (1976). Teacher involvement in curriculum planning. En J. Kane (ed.), Curriculum development in Physical Education (pp.97-121). London: Crosby Lockwood Staples.

Almond, L. (1979). The evaluation of teachers' practice in school. New Zealand Journal of Health, Physical Education, and Recreation, vol.12, n.3, 74-78.

Almond, L. (1985). Teaching games through action research. En Teaching Team Sports. International AIESEP Congress (pp.185-197). Roma: CONI.

Aquino, H., Orozco, M., Mendoza, M., Figueroa, M. y Orendain, C. (2008). Práctica docente de la educación física en la escuela primaria. Zapopan-Guadalajara: Instituto Superior de Investigación y Docencia del Magisterio.

Aquino, B., Rodríguez, C. y Aquino, H. (2001). Intervención reeducativa en el proceso de readaptación a los menores infractores de la Granja Juvenil de Readaptación Social a través de talleres físico deportivos. Guadalajara: ESEF JAL, Reporte de investigación interno, Unidad de Investigación de la ESEF JAL.

Aquino, H. (2004). La formación de investigadores en proyectos comunitarios deportivo-recreativos. Tesis de Doctorado en Educación. Doctorado Interinstitucional; Universidad La Salle, Universidad Pedagógica Nacional Unidad 141, Instituto Superior de Investigación y Docencia para el Magisterio y Centro de Investigaciones Pedagógicas y Sociales.

Bain, L. (1989). Interpretive and critical research in sport and physical education. Research Quarterly for Exercise and Sport, n.60, 21-24.

Bain, L. (1990). Physical education teacher education. En W.R. Houston (ed.), Handbook of Research on Teacher Education (pp.758-781). New York: Macmillan.

Barbero, J. (1993). Las redes de la cultura física. Aproximación genealógica al saber médico en educación física. En J. Barbero (ed.), Investigación alternativa en educación física (pp.7-30). Málaga: Unisport.

Barbero, J. (1994). Bases epistemológicas de la educación fisica escolar: saberes e ideologías imperantes. Comunicación presentada al II Simposium Internacional de Educación Física Escolar y Deporte de Alto Rendimiento. Las Palmas de Gran Canaria.

Barbero, J. (1996). Cultura profesional y curriculum (oculto) en educación física. Reflexiones sobre las (im)posibilidades de cambio. Revista de Educación, n.311, 13-45.

Beamish, R. (1982). A critical examination of the epistemological limitations of the positivist approach to comparative sport studies. En J. Pooley y C. Pooley (eds.), Proceedings of the Second International Seminar on Comparative Physical Education and Sport (pp.131-162) Halifax: Dalhousie University Printing Center.

Benn, T., Dagkas, S. y Jawad, H. (2011). Embodied faith: Islam, religious freedom and educational practices in physical education. Sport, Education and Society, vol.16, n.1, 17-34. 
Beltrán-Carillo, V., Devís-Devís, J., Peiró-Velert, C. y Brown, D. (2012). When physical activity participation promotes inactivity: Negative experiences of Spanish adolescents in Physical Education and sport. Youth and Society, vol.44, n.1, 1-25.

Bernstein, B. (1998). Pedagogía, control simbólico e identidad. Madrid. Morata.

Bracht, V. y Caparroz, F. (2009). El deporte como contenido de la educación física escolar: la perspectiva crítica de la educación física brasileña. En L. Martínez y R. Gómez (coords.), La educación física y el deporte en la edad escolar. El giro reflexivo en la enseñanza (pp.53-89). Buenos Aires: Miño y Dávila.

Bracht, V., Pires, R., García, S. y Sofiste, A. (2002). A prática pedagógica em educação física: a mudança a partir da pesquisa-ação. Revista Brasileira de Ciências do Esporte, vol.23, n.2, 9-29.

Branham, P. (2003). Boys, masculinities and PE', Sport, Education and Society, vol. 8, n.1, 57-71.

Brooker, R. y Macdonald, D. (1999). Did we hear you?: issues of student voice in a curriculum innovation. Journal of Curriculum Studies, vol.31, n.1, 83-97.

Brown, D. y Evans, J. (2004). Reproducing gender? Intergenerational links and the male PE teacher as a cultural conduit in teaching physical education. Journal of Teaching in Physical Education, n.23, 48-70.

Carr, W. y Kemmis, S. (1988). Teoría crítica de la enseñanza. La investigación-acción en la formación del profesorado. Tr. J.A. Bravo. Barcelona: Martínez Roca.

Chepyator-Thomson, J. y Ennis, C. (1997). Reproduction and resistance to the culture of femininity and masculinity in secondary school physical education. Research Quarterly for Exercise and Sport, vol.68, n.1, 89-99.

Clarke, G. (1996). Conforming and contesting with (a) difference: how lesbian students and teachers manage their identities. International Studies in Sociology of Education, vol.6, n.2, 191-209.

Clarke, G. (1998). Queering the pitch and coming out to play: lesbians in physical education and sport. Sport, Education and Society, vol.3, n.2, 145-60.

Crum, B. (1986). Concerning the quality of the development of knowledge in Sport Pedagogy. Journal of Teaching in Physical Education, n.5, 211-20.

Dadds, M. (2008). Empathic validity in practitioner research. Educational Action Research, vol. 16, n.2, 279-290.

Dagkas, S. y Benn, T. (2006). Young Muslim women's experiences of Islam and physical education in Greece and Britain: a comparative study. Sport, Education and Society, vol.11, n.1, 21-38.

Debusk, M. y Hellison, D. (1989). Implementing a physical education self-responsibility model for delinquency-prone youth. Journal of Teaching in Physical Education, n.8, 104-12.

Devís-Devís, J. (1993). Introducción crítica a la investigación positivista en la enseñanza de la educación física. En J. Barbero (coord.), Investigación alternativa en educación física (pp.3172). Málaga: Unisport.

Devís-Devís, J. (1996). Educación física, deporte y currículum. Madrid: Visor.

Devís-Devís, J. (1997). Policy, practice, and reconversion in Spanish educational reform: teaching and teacher education in physical education. The Curriculum Journal, vol.8, n.2, 213-30.

Devís-Devís, J. (2001). (ed.) La educación física, el deporte y la salud en el siglo XXI. Alcoi: Marfil.

Devís-Devís, J. (2006). Socially Critical Research Perspectives. En D. Kirk, D. Macdonald y M. O'sullivan (eds.), Handbook of Physical Education (pp.37-58). London: Sage.

Devís-Devís, J. y Antolín, L. (2001). Emociones, profesorado e innovación en la educación física: la revisión de un estudio de casos. En J. Devís-Devís (ed.), La educación física, el deporte y la salud en el siglo XXI (pp.61-85) Alcoi: Marfil.

Devís-Devís, J., Molina-Alventosa, Jp, Peiró-Velert, C. y Kirk, D. (2011). Selection of printed curriculum materials in physical education: recontextualising pedagogical knowledge. European Physical Education Review, vol.17, n.1, 19-34.

Devís-Devís, J., Perdiguero, E., Silvestre, A. y Peiró-Velert, C. (1994). La representación social del cuerpo en un grupo de estudiantes de educación física. En A. Clemente, G. Musitu y M. 
Gutiérrez (eds.), Intervención educativa y desarrollo humano (pp.147-155). Valencia: Set i Set ediciones.

Devís-Devís, J. y Sparkes, A. (1999). Burning the book: a biographical study of a pedagogically inspired identity crisis in physical education. European Physical Education Review, n.5, 135-52.

Devís-Devís, J. y Sparkes, A. (2004). La crisis de identidad de un estudiante universitario de educación física: La reconstrucción de un estudio biográfico. En A. Sicilia y J-M. FernándezBalboa (coords.), La otra cara de la investigación. Reflexiones desde la educación física (pp.83-108). Sevilla: Wanceulen.

Dewar, A. (1987). The social construction of gender in physical education. Women's Studies International Forum, n.10, 453-65.

Dewar, A. (1990). Oppression and privilege in physical education: struggles in the negotiation of gender in a university programme. En D. Kirk y R. Tinning (eds.), Physical Education, Curriculum and Culture (pp.67-99). London: The Falmer Press.

Dewar, A. (1993). Would all the generic women in sport please stand up? Challenges facing feminist sport sociology. Quest, n.45, 211-29.

Dowling, F. (2008). Getting in touch with our feelings: the emotional geographies of gender relations in PETE. Sport, Education and Society, vol.13, n.3, 247-266.

Ellsworth, E. (1989). Why doesn't this feel empowering? Working through the repressive myths of critical pedagogy. Harvard Educational Review, vol.59, n.3, 297-324.

Ennis, C. (1999). Creating a culturally relevant curriculum for disengaged girls. Sport, Education and Society, vol.4, n.1, 31-50.

Ennis, C., Cothran, D., Davidson, K., Loftus, S., Owens, L., Swanson, L. y Hopsicker, P. (1997). Implementing curriculum within a context of fear and disengagement. Journal of Teaching in Physical Education, n.17, 52-71.

Escartí, A., Pascual, C. y Gutiérrez, M. (2005). Responsabilidad personal y social a través de la educación física y el deporte. Barcelona: Graó.

Escartí, A., Gutiérrez, M., Pascual, C. y Marín, D. (2010). Application of Hellison's teaching personal and social responsibility model in physical education to improve self-efficacy for adolescents at risk of dropping-out of school. Spanish Journal of Psychology, vol.13, n.2, 667- 676.

Evans, J. (1986). (ed.) Physical education, sport and schooling. Studies in the sociology of physical education. London: The Falmer Press.

Evans, J. (1988). (ed.). Teachers, Teaching and Control in Physical Education. London: The Falmer Press.

Evans, J. (1993). (ed.). Equality, Education and Physical Education. London: The Falmer Press.

Evans, J. y Davies, B. (2011). (eds.) New directions, new questions? Social theory, education and embodiment. Sport, Education and Society (monograph).

Evans, J.; Davies, B. y Wright, J. (2004). (eds.) Body Control and Knowledge. Studies in the Sociology of Physical Education and Health. London: Routledge.

Fals-Borda, O. (1992). La ciencia y el pueblo: nuevas reflexiones. En M. C. Salazar (ed.), La investigación acción participativa. Inicios y desarrollos (pp.65-84). Madrid: Popular-OEIQuinto Centenario.

Fay, B. (1987). Critical social science. Liberation and its limits. Cambridge: Polity Press.

Fernández-Balboa, J. (1993). Aspecto crítico y cívico del rol de los/las profesionales de la educación física y el deporte: conexiones con la política, la economía y el medio ambiente. Apunts. Educació Física, n.34, 74-82.

Fernández-Balboa, J. (1997a) (ed.). Critical Postmodernism in Human Movement, Physical Education and Sport. Albany: SUNY.

Fernández-Balboa, J. (1997b). Physical education teacher preparation in the postmodern era: toward a critical pedagogy. En J. Fernández-Balboa, (ed.). Critical Postmodernism in Human Movement, Physical Education and Sport (pp.121-138) Albany: SUNY. 
Fitzgerald, H. (2005). Still feeling like a spare piece of luggage? Embodied experiences of (dis)ability in physical education and school sport. Physical Education and Sport Pedagogy, n.10, 41-59.

Fitzgerald, H., Jobling, A. y KIRK, D. (2003). Listening to the 'voices' of students with severe learning difficulties through a task-based approach to research and learning in physical education. Support for Learning, vol.18, n.3, 123-129.

Flintoff, A. (1994). Sexism and homophobia in physical education: the challenge for teacher educators. Physical Education Review, vol.17, n.2, 97-105.

Flintoff, A., Fitzgerald, H. Y Scraton, S. (2008). The challenges of intersectionality: researching difference in physical education. International Studies in Sociology of Education, vol.18, n.2, 73-85.

Foley, D. (1992). Making the familiar strange: writing critical sport narratives. Sociology of Sport Journal, vol.9, n.1, 36-47.

Fraile, A. (2004). (coord.). Didáctica de la educación física. Una perspectiva crítica y transversal. Madrid: Biblioteca Nueva.

Fraile, A. y ROMO, C. (1991). Investigación-acción en la educación física. Revista Interuniversitaria de Formación del Profesorado, n.10, 225-236.

Freire, P. (1973). Pedagogía del oprimido. 8th edition. Tr. J. Mellado. México: Siglo XXI.

Freire, P. (1987). L'educació com a pràctica de la llibertat. Tr. A. Roca. Vic: Eumo-Diputació de Barcelona.

Gage, N. (1989). The paradigm wars and their aftermath: A 'historical' sketch of research on teaching since 1989. Educational Researcher, vol.18, n.7, 4-10.

George, L. y Kirk, D. (1988). Limits of change in physical education: ideologies, teachers and the experience of physical activity. En J. Evans (ed.), Teachers, teaching and control in physical education (pp.15-155). London: The Falmer Press.

Goleman, D. (1999). Inteligencia emocional (29 edición). Barcelona: Kairós.

González-Ravé, J., Ruiz, L. y Carrasco, M. (2007). The social construction of gender in Spanish physical education students. Sport, Education and Society, vol.12, n2, 141-158.

Gore, J. (1992). What can we do for you! What can "we" do for "you"? Struggling over empowerment in critical and feminist pedagogy. En C. Luke y J. Gore (eds.), Feminisms and critical pedagogy (pp.54-73). London: Routledge.

Gore, J. (1996). Controversias entre las pedagogías. Tr. P. Manzano. Madrid: Morata.

Griffin, P. (1983). Gymnastics is a girl's thing: Student participation and interaction patterns in a middle school gymnastics unit. En T. Templin y J. Olson (eds.), Teaching in Physical Education (pp.71-75) Champaign: Human Kinetics.

Griffin, P. (1984). 'Girls' participation patterns in a middle school team sports unit. Journal of Teaching in Physical Education, n.4, 30-38.

Griffin, P. (1985a). Boys' participation styles in a middle school physical education team sports unit. Journal of Teaching in Physical Education, n.4, 100-10.

Griffin, P. (1985b) Teachers' perceptions of and responses to sex equity problems in a middle school physical education program. Research Quarterly for Exercise and Sport, n.56, 103-10.

Griffin, P. (1985c). Teaching in an urban, multiracial physical education program: the power of context. Quest, n.37, 154-65.

Griffin, P. (1992a). Identity management strategies among lesbian and gay educators. International Journal of Qualitative Studies in Education, vol.4, n.3, 189-202.

Griffin, P. (1992b). Changing the game: homophobia, sexism, and lesbians in sport. Quest, vol.44, n.2, 251-65.

Griffin, P. (1998). Strong Women, Deep Closets: Lesbians and Homophobia in Sports. Champaign: Human Kinetics.

Habermas, J. (1982). Conocimiento e interés. Tr. M. Jiménez, J.F. Ivars and L. Martín. Madrid: Taurus. Harris, J. (1987). Social contexts, scholarly inquiry, and physical education'. Quest, n.39, 282-94. 
Hay, P. y Macdonald, D. (2010). Evidence for the social construction of ability in physical education. Sport, Education and Society, vol.15, n.1, 1-18.

Hellison, D. (1978). Beyond Balls and Bats: Alienated (and other) Youth in the Gym. Washington: AAHPER.

Hellison, D. (1985). Goals and Strategies for Teaching Physical Education. Champaign: Human Kinetics.

Hellison, D. y Walsh, D. (2002). Responsibility-based youth programs evaluation: investigating the investigations. Quest, n.54, 292-307.

Hernández, J. (1996). La construcción histórica y social de la educación física: el currículo de la LOGSE, una nueva definición de la educación física escolar. Revista de Educación, n.311, 51-76.

Hickey, C. y Fitzclarence, L. (1999). Educating boys in sport and physical education: using narrative methods to develop pedagogies of responsibility. Sport, Education and Society, n.4, 51-62.

Hickey, C., Fitzclarence, L. y Matthews, R. (2000). (eds.) Where the boys are. Masculinity, sport and education. 2nd edn. Deakin: Deakin University Press.

Kemmis, S. (1992). L'educació del professor i el desenvolupament de comunitats crítiques de professors. En A. Sansano \& E. Contell (coords.), Crítica de la pedagogia i pedagogia crítica: textos per al debat. València: Galàxia d'Edicions-Federació de Moviments de Renovació Pedagògica.

Kincheloe, J. y Mclaren, P. (2000). Rethinking critical theory and qualitative research. En N. Denzin y Y. Lincoln (eds.), Handbook of Qualitative Research (pp.279-313) 2nd edn. Beverly Hills, CA: Sage.

Kirk, D. (1986). A critical pedagogy for teacher education: toward an inquiry-oriented approach. Journal of Teaching in Physical Education, n.5, 230-46.

Kirk, D. (1988). Physical Education and Curriculum Study. A Critical Introduction. London: Croom Helm.

Kirk, D. (1989). The orthodoxy in RT-PE and the research/practice gap: a critique and alternative view. Journal of Teaching in Physical Education, n.8, 123-30.

Kirk, D. (1990). Educación Física y Currículum. Universitat de València. Valencia.

Kirk, D. (1992). Defining Physical Education. The Social Construction of a School Subject in Postwar Britain. London: The Falmer Press.

Kirk, D. (1998a). Schooling Bodies. School Practice and Public Discourse, 1880-1950. London: Leicester University Press.

Kirk, D. (1998b). Educational reform, physical culture and the crisis of legitimation in physical education. Discourse: Studies in the Cultural Politics of Education, vol.19, n.1, 101-12.

Kirk, D. (2001). Fundamentos para una pedagogía crítica en la formación del profesorado de educación física. En J. Devís-Devís (ed.), La educación física, el deporte y la salud en el siglo XXI (pp.101-209). Alcoi: Marfil.

Kirk, D. y Colquhoun, D. (1989). Healthism and physical education. British Journal of Sociology of Education, vol.10, n.4, 417-34.

Kirk, D. y Smith, S. (1986). How objective are ROSBA objectives? A critique of objectivism in curriculum design. Curriculum Perspectives, vol.6, n.2, 32-36.

Kirk, D. y Tinning, R. (1990). (ed.) Physical Education, Curriculum and Culture: Critical Issues in the Contemporary Crisis. London: The Falmer Press.

Kirk, D. y Macdonald, D. (1998). Situated learning in physical education. Journal of Teaching in Physical Education, n.17: 376-87.

Kirk, D. y Macphail, A. (2002). Teaching games for understanding and situated learning: rethinking the Bunker-Thorpe model. Journal of Teaching in Physical Education, vol.21, n.2, 177-92.

Kirk, D., Mckay, J. y George, L. (1986). All work and no play? Hegemony in the P.E. curriculum. En Trends and Developments in P.E.: Proceedings of the VIII Commonwealth and International Conference on Sport, P.E., Dance, Recreation and Health (pp.170-177). London: E. and F.N. Spon. 
Klemola, U., Heikinaro-Johansson, P. y O'sullivan, M. (in press) Physical education student teachers' perceptions of applying knowledge and skills about emotional understanding studied in PETE in a one-year teaching practicum. Physical Education and Sport Pedagogy. DOI 10.1080/17408989.2011.630999.

Kunz, E. (1991) Educaçao física: ensino e mudanças. Ijuí: Unijuí.

Lake, J. Stratton, G.; Martin, D. y Money, M. (2001). Physical education and sustainable development: an untrodden path. Quest, n.53, 471-82.

Landau, G. (1996). Critical theory in German Sport Pedagogy. En P. Schempp (ed.), Scientific development of sport pedagogy (pp. 223-236), Münster: Waxman.

Lather, P. (1991). Getting Smart. Feminist Research and Pedagogy with/in the Postmodern. New York: Routledge.

Lawson, H. (1983a). New directions for research on teacher education and school practice in physical education. International Journal of Physical Education, 3-4: 10-15 and 8-14.

Lawson, H. A. (1983b). Paradigms for research on teaching and teachers. En T. Templin y J. Olson (eds.), Teaching in Physical Education. Champaign: Human Kinetics. (pp. 339-58.)

Lawson, H. (1984). Problem-setting for physical education and sport. Quest, n.36: 46-60.

Lawson, H. (1985). Knowledge for work in the physical education profession. Sociology of Sport Journal, n.2, 9-24.

Lawson, H. (1990a). Beyond positivism: research, practice, and undergraduate professional education. Quest, n.42, 161-83.

Lawson, H. (1990b). Sport Pedagogy research: from information-gathering to useful knowledge. Journal of Teaching in Physical Education, n.10, 1-20.

Lawson, H. (1991). Ideological, behavioral and structural facilitators for collaboration among researchers and practitioners. AIESEP-NAPEHE World Congress. Atlanta, Georgia.

Lawson, H. (1999). Education for social responsibility: preconditions in retrospect and in prospect. Quest, n.51, 116-49.

Light, R. y Kirk, D. (2000). High school rugby, the body and the reproduction of hegemonic masculinity. Sport, Education and Society, vol.5, n.2, 163-76.

López-Pastor, V. (2006). (coord.) Evaluación en educación física: Revisión de los modelos tradicionales y planteamiento de una alternativa: la evaluación formativa y compartida. Buenos Aires: Miño y Dávila.

López-Pastor, V., Monjas, R. y Manrique, J. (2011). Fifteen years of action research as professional development: seeking more collaborative, useful and democratic systems for teachers. Educational Action Research, vol.19, n.2, 153-170.

Macdonald, D. (2003). Curriculum change and the post-modern world: is the school curriculumreform movement and anachronism? Journal of Curriculum Studies, vol.35, n.2, 139-149.

Macdonald, D., Kirk, D. y Braiuka, S. (1999). The social construction of the physical activity field at the school/university interface. European Physical Education Review, vol.5, n.1, 31-51.

Macphail, A., Kirk, D. y Griffin, L. (2008) Throwing and catching as relational skills in game play: Situated learning in a modified game unit. Journal of Teaching in Physical Education, vol.27, n.1, 100-115.

Martínez, L. (1994) Influencia de las creencias implícitas en la formación inicial de los especialistas en educación física. En S. Romero (coord.), Didáctica de la educación física: diseños curriculares en primaria (225-232). Sevilla: Wanceulen.

Martínez, L. y Gómez, R. (2009). (coords.) La educación física y el deporte en la edad escolar. El giro reflexivo en la enseñanza. Buenos Aires: Miño y Dávila.

Mccaughtry, N. (2004). The emotional dimensions of a teacher's pedagogical content knowledge: influences of content, curriculum, and pedagogy. Journal of Teaching in Physical Education, n.23, 30-47. 
Mctaggart, R. (1997). Reading the collection. En R. McTaggart (ed.) Participatory Action Research, (pp. 1-24), Albany: SUNY.

Molina, J. y Beltrán, V. (2007). Incompetencia motriz e ideología del rendimiento en educación física: El caso de un alumno con discapacidad intelectual. Motricidad. European Journal of Human Movement, n.19, 165-190.

Molina, J. y Devís-Devís, J. (2001). La educación física en la reforma educativa actual: análisis crítico. En B. Vázquez (coord.) Bases educativas de la actividad física y el deporte (pp.301331). Madrid: Síntesis.

Molina-Neto, V. (1996). La cultura docente del profesorado de educación física de las escuelas públicas de Porto Alegre. Tesis doctoral inédita $(\mathrm{PhD})$. Universitat de Barcelona, Barcelona.

Molina-Neto, V. (2003). Crenças do professorado de Educação Física das escolas públicas de Porto Alegre - RS/Brasil. Movimento, vol.9, n.1, 145-169.

Muñoz, G. (2004). Planejamento coletivo do trabalho pedagógico da Educação Física - PCTP/ EF como sistemática de formação continuada de professores: a experiência de Uberlândia. Movimento, vol.10. n.1, 113-131.

Murcia, N., Jaramillo, L., Camacho, H. y Loaiza, M. (2005). Imaginarios de los jóvenes ante la clase de Educación Física. Armenia: Kinesis.

Muros, B. y Fernández-Balboa, J. (2005). Physical education teacher educator's personal perspectives regarding their practice of critical pedagogy. Journal of Teaching in Physical Education, vol.24, n.3, 243-264.

O'sullivan, M. y Macphail, A. (2010). (eds.) Young people's voices in physical education and youyh sport. Abingdon: Routledge.

O'sullivan, M., Siedentop, D. y Locke, L. (1992). Toward collegiallity: competing viewpoints among teacher educators. Quest, n.44, 266-80.

Paechter, C. (2003). Masculinities and fmeninities as communities of practice. Women's Studies International Forum, vol.26, n.1, 69-77.

Peiró-Velert, C. y Devís-Devís, J. (1993). Innovación en educación física y salud: el estudio de un caso en investigación colaborativa. En J. Barbero (ed.), Investigación alternativa en educación física (pp.249-270). Málaga: Unisport.

Penny, D. y Evans, J. (1999). Politics, Policy and Practice in Physcial Education. London: E \& FN Spon.

Pérez-Samaniego, V., Devís-Devís, J., Smith, B. y Sparkes, A. (2011a). La investigación narrativa en la educación física y el deporte: qué es y para qué sirve. Movimento, vol.17, n.1, 11-38

Pérez-Samaniego, V., Fuentes-Miguel, J. y Devís-Devís, J. (2011b). El análisis narrativo en la educación física y el deporte. Movimento, vol.17, n.4, 11-42.

Renold, E. (1997).All they've got on their brains is football'. Sport, masculinity and the gendered practice of the playground relations. Sport, Education and Society, vol.2, n.1, 5-23.

Rovegno, I. y Kirk, D. (1995). Articulations and silences in socially critical work on physical education: toward a broader agenda. Quest, n.47, 447-74.

Ruiz, E., López, C. y Escobar, J. (2011). Los jóvenes, el ideal estético y la televisión. El cuerpo real y el imaginado. Revista Luciérnaga, vol.3, n.6, 17-22.

Schempp, P. (1987). Research on teaching physical education: Beyond the limits of natural science. Journal of Teaching in Physical Education, n.6, 111-21.

Schempp, P. (1988). Exorcist II: a reply to Siedentop. Journal of Teaching in Physical Education, n.7, 79-81.

Scraton, S. (1986). Images of femininity and the teaching of girls' physical education. En John Evans (ed.), Physical Education, Sport and Schooling. Studies in the Sociology of Physical Education (pp.71-94). London: The Falmer Press. 
Scraton, S. (1987). Boys muscle in where angels fear to tread' -girls' subcultures and physical activities. En J. Horn, D. Jary and A. Thomlinson (eds.), Sport, Leisure and Social Relations (pp.160-186). London: Routledge and Keagan Paul.

Scraton, S. (1995). La educación física de las niñas: un enfoque feminista. Madrid: Morata.

Sicilia, A. (2011). (coord.) La evaluación y calificación en la universidad. Relatos autobiográficos durante la búsqueda de alternativas. Barcelona: Hipatia.

Sicilia, Á. y Fernández-Balboa, J. (2004). (eds.) La otra cara de la investigación. Reflexiones desde la educación física. Sevilla: Wanceulen.

Sicilia-Camacho, A. y Fernández-Balboa J. (2009). Reflecting on the moral bases of critical pedagogy. En PETE: toward a Foucaultian perspective on ethics and the care of the self. Sport, Education and Society, vol.14, n.4, 443-463

Siedentop, D. (1987). Dialogue or exorcism? A rejoinder to Schempp. Journal of Teaching in Physical Education, n.6, 373-376.

Silverman, S. (1991). Research on teaching in physical education. Research Quarterly for Exercise and Sport, vol.62, n.4, 352-364.

Sirna, K., Tinning, R. y Rossi, T. (2008). The social tasks of learning to become a physical education teacher: Considering the HPE subject department as a community of practice. Sport, Education and Society, vol.13, n.3, 285-300.

Smyth, J. y Shacklock, G. (1998). Behind the 'Cleansing' of socially critical research accounts. En G. Shacklock y J. Smyth (eds.), Being Reflexive in Critical Educational and Social Research (pp.1-12). London: The Falmer Press.

Soler, S. (2009). Los procesos de reproducción, resistencia y cambio de las relaciones tradicionales de género en la educación física: el caso del fútbol. Cultura y Educación, vol.21, n.1, 31-42.

Sparkes, A. (1988a). The micropolitics of innovation in the physical education curriculum. En J. Evans (ed.), Teachers, Teaching and Control in Physical Education. London: The Falmer Press. (pp.157-77)

Sparkes, A. (1988b). Strands of commitment within the process of teacher initiated innovation. Educational Review, vol.40, n.3, 301-17.

Sparkes, A. (1989). Towards an understanding of the personal costs and rewards involved in teacher initiated innovations. Educational Management and Administration, n.17, 100-208.

Sparkes, A. (1992). (ed.) Research in Physical Education and Sport. Exploring Alternative Visions. London: The Falmer Press.

Sparkes, A. (1994a). Self, silence and invisibility as a beginning teacher: a life history of lesbian experience. British Journal of Sociology of Education, vol.15, n.1, 93-118.

Sparkes, A. (1994b). Life histories and the issue of voice: reflections on an emerging relationship. International Journal of Qualitative Studies in Education, vol.7, n.2, 165-83.

Sparkes, A. (1997). Ethnographic fiction and representing the absent other. Sport, Education and Society, vol.2, n.1, 25-40.

Sparkes, A. (2002a). Telling tales in sport and physical activity: a qualitative journey. Champaign: Human Kinetics.

Sparkes, A. (2002b). Fictional representations: on difference, choice, and risk. Sociology of Sport Journal, vol.19, n.1, 1-24.

Sparkes, A. (2009). Novel ethnographic representations and the dilemmas of judgement. Ethnography and Education, vol.4, n.3, 301-319.

Sparkes, A. y Devís- Devís, J. (2007). La investigación narrativa y sus formas de análisis: una visión desde la educación física y el deporte. En W. Moreno y S.M. Pulido (eds.), Educación cuerpo y ciudad: el cuerpo en las interacciones e instituciones sociales (pp.43-68). Medellin: Funámbulos.

Sparkes, A., Nilges, L., Swan, P. y Dowling, F. (2003). Poetic Representations in Sport and Physical Education: Insider Perspectives 1. Sport, Education and Society, vol.8, n.2, 153-177. 
Sparkes, A. y Smith, B. (2008). Men, spinal cord injury, memories and the narrative performance of pain. Disability and Society, vol.23, n.7, 679-690.

Sparkes, A., Templin, T. y Schempp, P. (1990). The problematic nature of a career in a marginal subject: some implications for teacher education programmes. Journal of Education for Teaching, vol.16, n.1, 3-27.

Sparkes, A., Templin, T. y Schempp, P. (1993). Exploring dimensions of marginality: reflecting on the life histories of physical education teachers. Journal of Teaching in Physical Education, vol.12, n.4, 386-98.

Squires, S. y Sparkes, A. (1996). Circles of silence: sexual identity in physical education and sport. Sport, Education and Society, n.1, 77-102.

Steinhardt, M. (1992). Physical education. En P. Jackson (ed.), Handbook of Research on Curriculum (pp.964-1001) New York: Macmillan.

Sykes, H. (2001). Understanding and overstanding: feminist.poststructural life histories of physical education teachers. Qualitative Studies in Education, vol.14, n.1, 13-31.

Tinning, R. (1985). Physical education and the cult of slenderness. A critique. The ACHPERD National Journal, n.107, 10-13.

Tinning, R. (1987a). Improving Teaching in Physical Education. Deakin: Deakin University Press.

Tinning, R. (1987b). Beyond the development of a utilitarian teaching perspective: an australian case study of action research in teacher preparation. En G. Barrette, R. Feingold, C. Rees \& M. Piéron (eds.), Myths, Models and Methods in Sport Pedagogy (pp.113-122). Champaign: Human Kinetics.

Tinning, R. (1988). Student teaching and the pedagogy of necessity. Journal of Teaching in Physical Education, vol.7, n.2, 82-89.

Tinning, R. (1991). Teacher education pedagogy: dominant discourses and the process of problem solving. Journal of Teaching in Physical Education, vol.11, n.1, 1-20.

Tinning, R. (1992a). Action research as epistemology and practice: towards transformative educational practice in physical education. En A. Sparkes (ed.), Research in Physical Education and Sport. Exploring Alternative Visions (pp.188-209). London: The Falmer Press.

Tinning, R. (1992b). Educación física: la escuela y sus profesores. Universitat de València. Valencia.

Tinning, R. (1997). Performance and participation discourses in Human Movement: toward a socially critical physical education. En J. Fernández-Balboa (ed.), Critical Postmodernism in Human Movement, Physical Education and Sport (pp.99-119). Albany: SUNY.

Tinning, R. (2002). Toward a "Modest pedagogy": reflections on the problematics of critical pedagogy. Quest, n.54, 224-40.

Tinning, R. y Fitzclarence, L. (1992). Postmodern youth culture and the crisis in Australian secondary school physical education. Quest, n.44, 287-303.

Tinning, R.; Macdonald, D.; Tregenza, K. y Boustead, J. (1996) Action research and the professional development of teachers in the health and physical education field: the Australian NPDP experience. Journal of Educational Action Research, 4 (3): 389-405.

Trigueros, C. Rivera, E. y De La Torre, E. (2006) El aprendizaje colaborativo en la formación de maestros. Una experiencia práctica. Tándem. Didáctica de la Educación Física, n.20, 45-55.

Wright, J. (1990). Remember those skirts!: the constitution of gender in physical education. En S. Scraton (ed.), Gender and physical education (pp.63-77) Deakin: Deakin Unversity Press.

Wright, J. (1995). A feminist poststructuralist methodology for the study of gender construction in physical education: description of study. Journal of Teaching in Physical Education, n. 15, 1-24.

Wright, J. (1997). The construction of gendered contexts in single sex and co-educational physical education lessons. Sport, Education and Society, vol.2, n.1, 55-72.

Wright, J. (2000). Bodies, meanings and movement: a comparison of the language of physical education lesson and a Feldenkrais movement class. Sport, Education and Society, vol.5, n.1, 35-50. 
Wright, J. (2004). Post-structural methodologies: the body, schooling and health. En J. Evans, B. Davies y J. Wright (eds.), Body Control and Knowledge. Studies in the Sociology of Physical Education and Health (pp.19-31) London: Routledge.

Wright, J., Macdonald, D. y Groom, L. (2003). Physical activity and young people: beyond participation. Sport, Education and Society, vol.8, n.1, 17-33.

Wright, P.M., White, K. y Gaebler-Spira, D. (2004). Exploring the relevance of the personal and social responsibility model in adapted physical activity: a collective case study. Journal of Teaching in Physical Education, n.23: 71-87.

Zamosc, L. (1992). Campesinos y sociólogos: reflexiones sobre dos experiencias de investigación activa. En M. Salazar (ed.), La investigación acción participativa. Inicios y desarrollos (pp. 85-134). Madrid: Popular-OEI-Quinto Centenario. 
